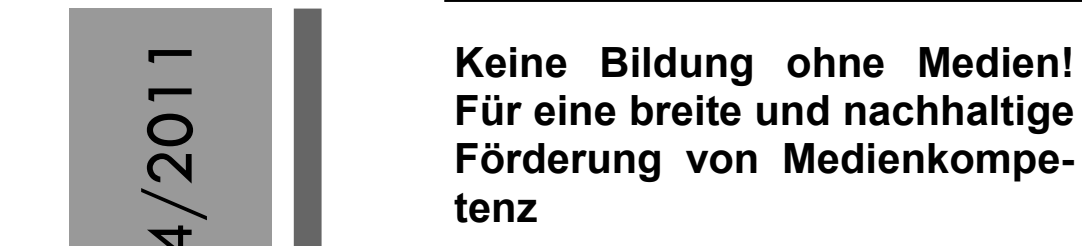

Einführungsvortrag auf dem Medienpädagogischen Kongress am 24. März 2011 an der TU Berlin

\section{HORST NIESYTO}

Die Initiative „Keine Bildung ohne Medien!“ freut sich über die starke Resonanz auf diesen Kongress. Die Resonanz zeigt: Nicht nur die Netzwerkwerke funktionieren gut. In vielen Rückmeldungen, die mich in den letzten Wochen erreichten, wurde deutlich: Es gibt die Erwartung, dass von dem medienpädagogischen Kongress ein wichtiger Impuls zu einer breitenwirksamen und nachbaltigen Verankerung von Medienpädagogik und Medienbildung in Deutschland ausgeht!

Wer die „Bildungsrepublik Deutschland“ will, wer wirklich will, dass Bildung Priorität hat, der muss Medienkompetenz und Medienbildung ganz anders fördern als bisher. Deshalb fordern wir: Keine Bildung ohne Medien!

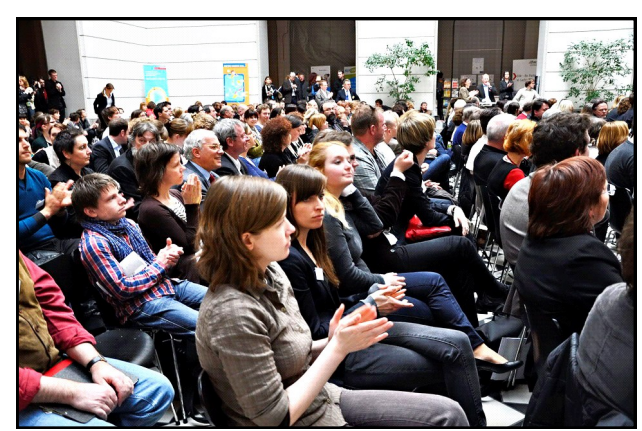

Foto: Vanessa Dreischer-Scheib

Erinnern wir uns: Das Medienpädagogische Manifest wurde im Frühjahr 2009 veröffentlicht. Obgleich es in der Vergangenheit immer wieder Kooperationen zwischen medienpädagogischen Einrichtungen und Organisationen gab, so fehlte es doch an einer übergreifenden Initiative, um gemeinsam bildungspolitische Vorstellungen in der Öffentlichkeit zu artikulieren. Es ist sehr positiv zu bewerten, dass es gelang, sich auf eine gemeinsame Einschätzung der Situation und zentrale Forderungen zu verständigen. Ich bedanke mich hier noch einmal bei den Kolleginnen und Kollegen der Erstunterzeichner-Organisationen für ihre Bereitschaft, diesen wichtigen Schritt gemacht zu haben:

- der Gesellschaft für Medienpädagogik und Kommunikationskultur, GMK

- dem JFF - Institut für Medienpädagogik in Forschung und Praxis

- dem Hans-Bredow-Institut für Medienforschung

- den Kolleginnen und Kollegen in der Sektion Medienpädagogik in der Deutschen Gesellschaft für Erziehungswissenschaft

- und der Fachgruppe Medienpädagogik in der Deutschen Gesellschaft für Publizistik und Kommunikationswissenschaft.

Im Medienpädagogischen Manifest haben wir geschrieben:

„Die Verschmelzung der alten und der neuen Medien, ihre zeit- und ortsunabhängige Verfügbarkeit sowie der Zugriff zum Internet eröffnen den Menschen neue Lern- und Erfahrungsbereiche. Medien bieten Möglichkeiten zur Selbstverwirklichung und zur kulturellen und gesellschaftlichen Teilhabe. Darüber hinaus liefern Medien wichtige Deutungsangebote, Identifikations-, Orientierungs- und Handlungsräume. Sie sind eine kontinuierlich verfügbare Ressource für Identitätskonstruktionen von Heranwachsenden. Gleichzeitig bringen sie auch neue Entwicklungs- und Sozialisationsprobleme sowie gesellschaftliche Risiken mit sich. Diese reichen von ethisch fragwürdigen Medienangeboten über soziale Benachteiligung bis hin zu fahrlässigen Formen des Umgangs mit (digitalen) Medien.“

Wir haben daraus geschlussfolgert, dass „die sozialen und kulturellen Auswirkungen globalisierter Medienwelten und die Entwicklung der Gesellschaft zu einer allumfassenden Informations- und Mediengesellschaft (...) den gesamten Bildungsbereich und damit auch die Medienpädagogik auf neue Weise" herausfordern. Es bedarf großer Anstrengungen, um die Menschen zu unterstützen, diesen technologischen Wandel zu verarbeiten und Medien aktiv, kreativ und verantwortungsvoll zu nutzen. Der Umgang mit den vielfältigen Medienangeboten und die eigene Artikulation mittels Medien benötigt Kompetenzen. Diese Kompetenzen reichen u. a. von einer Informationskompetenz zur kritischen Quellenund Qualitätsbewertung, Hintergrundwissen zur Machart von Medienproduktionen, eigenen Kriterien für die Auswahl und Nutzung von Medienangeboten über Kompetenzen zur Gestaltung, Produktion, zur Kommunika- 
tion und zum Lernen mit Medien bis hin zu Fragen von Persönlichkeitsrechten und des medienerzieherischen und medienethischen Handelns in der Gesellschaft.

Bildungsprozesse mit Medienbezug setzen dabei am vorhandenen Mediengebrauch der Menschen an und intendieren die Förderung eines medienkompetenten Handelns. Die Ressourcen für einen kompetenten Mediengebrauch, insbesondere die sozialen und bildungsbezogenen Ressourcen, sind in dieser Gesellschaft sehr unterschiedlich verteilt. Medienpädagogik ist gefordert, Gegengewichte zu Prozessen gesellschaftlicher Ausgrenzung zu setzen, um Menschen aus bildungsbenachteiligten Milieus Erfahrungen der Selbstwirksamkeit durch handlungsorientierte Formen der Medienbildung zu ermöglichen. Vor allem in der praktischen Auseinandersetzung mit Medien kann es gelingen, Fähigkeiten zur reflexiven Distanz gegenüber medialen Inszenierungen auszubilden. Dabei ist beachten: Soziokulturelle Unterschiede in der Mediennutzung verweisen nicht automatisch auf Aspekte sozialer Benachteiligung, sondern zunächst einmal auf andere medien- und sozial-ästhetische Muster und Präferenzen. Formen sozialer Benachteiligung in der Medienaneignung werden vor allem dann sichtbar, wenn Anregungen in Familie, Freundeskreis oder Schule nicht ausreichen, um Medien aktiv und reflexiv zu nutzen. In dieser Situation ist es erforderlich, Angebote zur Medienkompetenzförderung erheblich auszubauen und lebensweltnah mit Förderaufgaben in anderen Bereichen zu verknüpfen.

Im Hinblick auf alle Sozialgruppen ist festzuhalten: Es gibt keinen bestimmten Ort oder eine bestimmte Altersspanne, auf die Medienbildung zu konzentrieren wäre. Es sind alle Bildungsbereiche und alle Lebensphasen zu berücksichtigen. $\mathrm{Zu}$ diesen Bildungsbereichen gehört auch das Internet mit seinen social communities als neuer Ort für Information, Kommunikation und Bildung. Und es sind die Zusammenhänge zwischen den Lebensorten und Handlungsfeldern zu beachten. Medienbildung ist ein integraler Bestandteil unterschiedlicher Anforderungssituationen und lebenslanger Bildungsprozesse - nicht zuletzt auf dem Hintergrund der sich ständig weiter entwickelnden Medienumwelten.

Angesichts dieser Aufgaben ist die Frage, ob die bisherigen Ansätze und Aktivitäten ausreichen. Im Manifest wurde hierzu festgestellt:

„Die Medienpädagogik hat in den beiden vergangenen Jahrzehnten beachtliche Fortschritte in Theorie, Forschung und Praxis erzielt. So konnte eine Reihe notwendiger, aber längst nicht hinreichender medienpädagogischer Fundamente geschaffen werden: Theoretische und empirische Arbeiten beleuchten die viel- fältigen Dimensionen des Medienhandelns und die Bedeutung der Medien für Sozialisation und kulturelle Alltagspraktiken. Es gibt eine Fülle an hervorragenden medienpädagogischen Materialien für die Praxis, eine Vielzahl an überzeugenden Modellversuchen und eindrucksvollen Leuchtturmprojekten - aber es fehlt an der erforderlichen Nachbaltigkeit. Es mangelt nach wie vor an der Infrastruktur und an den organisatorischen Rahmenbedingungen in den Bildungseinrichtungen sowie an der medienpädagogischen Qualifikation der pädagogischen Fachkräfte.“

Auf dem Hintergrund dieser Einschätzung fordert das Manifest eine umfassende Förderung der Medienpädagogik, eine auf Jahre angelegte strategische Planung und insbesondere auch personelle, infrastrukturelle und finanzielle Investitionen auf Länder- und Bundesebene. Punktuelle Maßnahmen, neue Projektausschreibungen und diverse Informations- und Beratungsangebote im Internet reichen längst nicht mehr aus. Die zentrale Aufgabe besteht heute darin, die Medienpädagogik von einer Phase der Modellprojekte und einzelnen Aktionen auf lokaler und regionaler Ebene zu einer Phase struktureller Veränderungen zu überführen.

Mit besonderer Dringlichkeit stellt das Manifest folgende Forderungen auf:

1) Damit alle Kinder und Jugendlichen die Chance erhalten, ihre Medienkompetenzen zu erweitern, müssen medienpädagogische Programme vor allem in den Einrichtungen der Elementarpädagogik sowie in der Jugend-, Familien- und Elternbildung verstärkt werden.

2) Im schulischen Bereich sind für alle Schulformen Bildungsstandards für Medienkompetenz zu vereinbaren und entsprechende medienpädagogische Inhalte in Curricula verbindlich zu verankern, ergänzt um regelmäßige Evaluationsstudien, Programme zur Qualitätssicherung und nachhaltige Fortbildungsmaßnahmen für alle Lehrpersonen.

3) Einen besonderer Schwerpunkt ist auf Angebote für Kinder und Jugendliche aus Migrationskontexten und bildungsbenachteiligten Milieus sowie auf Angebote zur geschlechtersensiblen Arbeit zu legen. Dafür ist vor allem in der außerschulischen Kinder- und Jugendarbeit und in der kulturellen Bildung eine erhebliche Verbesserung der Infrastruktur und der personellen Ausstattung durch öffentliche Mittel zu gewährleisten.

4) In der Ausbildung von Erzieher/innen, Lehrer/innen, Erwachsenenbildnern/ innen und Sozialpädagogen/innen ist eine medienpädagogische Grundbildung als verbindlicher Bestandteil der pädagogi- 
schen Ausbildung zu verankern. Spezifische medienpädagogische Ausbildungen sind in Master-Studiengängen und als Wahlpflichtbereiche in anderen Studiengängen anzubieten. Dafür sind mehr medienpädagogischer Professuren und Lehrstühle mit Infrastruktur an den Hochschulen notwendig.

5) In der medienpädagogischen Forschung sind Forschungsprogramme zu etablieren, um insbesondere die Grundlagenforschung, die Mediensozialisationsforschung und die medienpädagogischen Begleit- und Praxisforschung deutlich zu stärken.

Das Medienpädagogische Manifest wurde von über

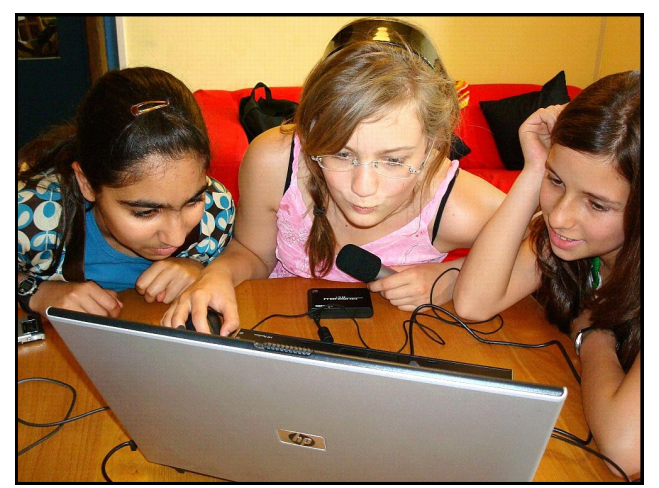

Foto: Verena Ketter

1300 Personen und Einrichtungen unterzeichnet, darunter auch mehrere Dachverbände und Organisationen auf Bundesebene. Gemessen an manch anderen Petitionen und Erklärungen mag diese Zahl als nicht so groß erscheinen. Aber bei dem Manifest geht es nicht um eine singuläre Forderung, sondern um ein ganzes Programm. Ich danke jedem, der unsere Anliegen und Forderungen mit seiner Unterschrift unterstützt hat! Auch möchte ich betonen, dass das Manifest sicherlich nicht alle Themen angesprochen hat, die für viele Kolleginnen und Kollegen auch wichtig sind. Um so mehr ist es zu schätzen, dass viele Personen unterschrieben haben, weil sie die Grundanliegen des Manifests teilen.

In der Öffentlichkeit wächst das Bewusstsein, dass in einer mediatisierten Gesellschaft die breite und nachhaltige Förderung von Medienkompetenz zu den unverzichtbaren Bildungsaufgaben gehört. Der enorme Wandel der technologischen Kommunikation verändert umfassend soziale Verhältnisse, Bildung und Kultur und hat Auswirkungen auf politische und wirtschaftliche Strukturen. Aktuell haben wir es wieder am Beispiel der gesellschaftlichen Umwälzungen im arabischen Raum oder bei dem Nachweis der Plagiatsvorwürfe gegenüber dem ehemaligen Verteidigungsminister zu Guttenberg erlebt, welche wichtige Bedeutung Internet, mobile Kommunikation und digitale Netzwerke für die Artikulation zivilgesellschaftlicher Interessen haben. Gleichzeitig verbinden sich mit der Medienentwicklung Problemfelder, die von einer starken Kommerzialisierung sozialer Kommunikation, risikobehafteten Nutzungen im Internet bis hin zum sog. digital divide reichen.

Die Medienpädagogik reagierte auf die Veränderung der Informations- und Kommunikationsverhältnisse und entwickelte diverse Angebote und Projekte. Auch außerhalb des Kernbereichs der Medienpädagogik engagieren sich viele Fachkräfte in Initiativen und Organisationen wie z. B. in der Gesellschaft für Medien in der Wissenschaft, dem Deutschen Bibliotheksverband, der Initiative D 21, der Deutschen Gesellschaft für Informationswissenschaft und Informationspraxis oder bei $e d u-$ camp, die sich mit der Entwicklung digitaler Medien und ihrer Bedeutung für Bildung, Wissenschaft und Wirtschaft befassen. Die Landesmedienanstalten führen zahlreiche Förderprojekte durch, die öffentlich-rechtlichen Rundfunk- und Fernsehanstalten engagieren sich seit vielen Jahren mit Informations- und Bildungsangeboten in unterschiedlichen Formaten. Auch neue Unternehmen im Bereich der Medien- und Kommunikationswirtschaft bieten vermehrt Online-Portale für Kinder und Jugendliche an; Buch-, Schulbuch-, Zeitungs- und Zeitschriftenverlage greifen verstärkt medienbezogene Bildungsthemen auf. In den letzten Jahren entstanden auch Unternehmen insbesondere im Bereich ComputerLernsoftware, die pädagogisch relevante Dienstleistungen und Produkte offerieren. Auch die Verbraucherbildung unternimmt in letzter Zeit vermehrt Anstrengungen, den Bereich Medienkompetenz in ihr Beratungsprofil zu integrieren.

Auf Bundesebene sind Fachabteilungen in Ministerien bemüht, den medientechnologischen Wandel unter Bildungsaspekten aufzugreifen. So setzte z. B. das Bundesministerium für Bildung und Forschung eine Expertenkommission ein, die 2009 den Bericht Kompetenzen in einer digital geprägten Kultur erarbeitete. Das Bundesbildungs- und das Bundesjugend- und -familienministerium luden 2010 und 2011 zu Fachkonferenzen ein, um Fragen der Medienkompetenzförderung und der Medienbildung zu erörtern. Im Deutschen Bundestag konstituierte sich die Enquetekommission Internet und digitale Gesellschaft und bildete eine spezielle Projektgruppe zum Thema Medienkompetenz. Die Gemischte Kommission der Kultusministerkonferenz beabsichtigt eine Aktualisierung der Erklärung Medienpädagogik in der Schule aus dem Jahre 1995. Die Länderkonferenz Medienbildung legte Positionspapiere vor, um auf eine bessere und nachhal- 
tige Verankerung von Medienbildung in Schule und Unterricht hinzuwirken.

Obgleich diese u. a. Entwicklungen zeigen,

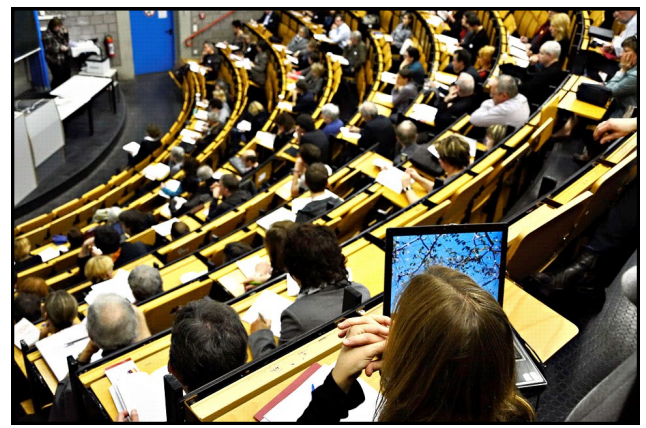

Foto: Vanessa Dreischer-Scheib

dass in letzter Zeit deutlich mehr Bewegung in die öffentliche Diskussion über die Notwendigkeit einer Medienkompetenzförderung gekommen ist, so fehlen nach wie vor breitenwirksame Maßnahmen, die über die bisherige Förderstrukturen deutlich hinausgehen. Zwar haben Bestrebungen zugenommen, vorhandene Angebote besser sichtbar zu machen und zu vernetzen. Medienbildung hat aber immer noch keine Priorität in den bildungspolitischen Beschlüssen. So kündigte z. B. die Bundesregierung in ihrer Koalitionsvereinbarung 2009 an, dass sie die Lehrerausbildung an deutschen Hochschulen stärken möchte: „Der Erhöhung der Medienkompetenz kommt dabei eine besondere Rolle zu“. Auf entsprechende Maßnahmen wird seither vergeblich gewartet; offensichtlich sind ursprünglich geplante Förderaktionen in Zusammenhang mit dem sog. „Sparhaushalt" gestrichen worden. Die politischen Prioritäten werden nach wie vor anders gesetzt, es existiert nach wie vor eine Kluft zwischen allgemeinen bildungspolitischen Proklamationen und tatsächlich breitenwirksamen Maßnahmen.

Mit Blick auf die Situation in verschiedenen Bundesländern und Handlungsfeldern ist diese Einschätzung sicherlich zu differenzieren. Hier gibt es teilweise Bestrebungen, die stärker in Richtung Nachhaltigkeit gehen und darauf hoffen lassen, dass sich in den nächsten Jahren mehr bewegt. Aktuell zu nennen sind u. a. das „10-Punkte-Programm“ der Landesregierung in Rheinland-Pfalz oder die Ankündigung in der Koalitionserklärung der Landesregierung in Nordrhein-Westfalen, NRW zum „Medienkompetenzland Nummer $1^{\text {" }}$ in Deutschland zu machen. An dieser Stelle ist auch eine positive Nachricht aus BadenWürttemberg zu vermelden: Im Rahmen der Neustrukturierung der Lehramtsausbildung an den Pädagogischen Hochschulen wurde festgelegt, dass künftig medienpädagogische Themen ein verbindlicher Bestandteil der mündlichen Abschlussprüfung in allen Fächern sind. Wenn es hier zu einem produktiven Wettbe- werb um die besten Konzepte und wirkungsvolle Fördermaßnahmen zwischen den Bundesländern kommt, ist dies zu begrüßen. Die Initiative „Keine Bildung ohne Medien!“ geht davon aus, dass neben bundesweiten Rahmenbedingungen vor allem auf regionaler und lokaler Ebene entscheidende Fortschritte zu erzielen sind.

Die Verbesserung struktureller Rahmenbedingungen kann dabei nicht unabhängig von den inhaltlichen Konzepten betrachtet werden. Ich sehe hier zwei unterschiedliche Entwicklungspfade in der Medienkompetenzförderung.

Der eine Entwicklungspfad betont vor allem anwendungsbezogenes Bedien- und Regelwissen im Umgang mit Medien. Entsprechende Kompetenzprofile sind oft sehr technikorientiert, zielen vor allem auf Effektivität und wirtschaftliche Wettbewerbsfähigkeit und unterschätzen die Bedeutung von (digitalen) Medien für Kommunikation, Orientierung und Identitätsbildung. Ein Denken, das nach wie vor vom Primat wirtschaftlicher Interessen und stetiger ökonomischer Wachstumsszenarien ausgeht und hierunter das „Humankapital“ zu- und unterordnet, konfligiert grundsätzlich mit einem Verständnis von Bildung und Medienbildung, welches die Menschen mit ihren unterschiedlichen Lebensbedürfnissen in den Mittelpunkt stellt. Medienkompetenzförderung hat die Chance, die Orientierungs-, Kommunikations- und Teilhabefähigkeit der Menschen in der Gesellschaft entscheidend $\mathrm{zu}$ erweitern. Sie verfehlt jedoch ihre sozialen und kulturellen Dimensionen, wenn sie Medienkompetenz primär in einem ökonomischen Verwertungszusammenhang verortet.

Dem anderen Entwicklungspfad liegt ein umfassendes Verständnis von Medienbildung zugrunde. Neben technischen und arbeitsweltbezogenen Kompetenzen rücken soziale, ästhetische, kulturelle und kommunikative Dimensionen mehr ins Blickfeld. Betont werden nicht engmaschig von Experten gestrickte Medienkompetenzniveaus, sondern die subjektorientierte und prozesshafte Entwicklung von medienbezogenen Bildungs- und Lernanlässen in sozialen Kontexten. Es geht um eine lebensweltorientierte Medienbildung. Der Expertenbericht des Bundesbildungsministeriums Kompetenzen in einer digital geprägten Kultur ist in diesem $\mathrm{Zu}$ sammenhang ein wichtiges Dokument. Der Bericht begründet die Notwendigkeit einer umfassenden Sicht auf Medienbildung für die Persönlichkeitsentwicklung, für die gesellschaftliche Teilhabe und für die Entwicklung von Ausbildungs- und Erwerbsfähigkeit. Er fokussiert nicht allein auf arbeitsweltrelevante Anforderungen, sondern betont vier Aufgaben - und Themenfelder: Information und Wissen, Kommunikation und Kooperation, Identitätssuche und Orientierung, digitale Wirklichkei- 
ten und produktives Handeln. Bei den Überlegungen zur Umsetzung dieser Aufgabenfelder empfiehlt der Bericht, an den Kompetenzen und Stärken anzusetzen, die junge Menschen in ihrem Alltag entwickeln, und diese als wichtige Ressourcen in Bildungsprozesse zu integrieren.

Wenn ich die Positionspapiere der Arbeitsgruppen sehe, die zu diesem Kongress vorgelegt wurden, habe ich den Eindruck, dass sich alle Papiere an dem skizzierten, umfassenden Verständnis von Medienbildung und Medienkompetenzförderung orientieren. Dies bedeutet nicht, arbeitswelt- und berufsbezogene Qualifikationen gering zu schätzen. Ich teile auch die Einschätzung, dass sich der Ansatz einer umfassenden Medienbildung und das Konzept eines Computer- und Medienführerscheins nicht grundsätzlich konträr gegenüberstehen, wenn Medienkompetenz nicht auf instrumentelle Fertigkeiten und Fähigkeiten verengt und ein Zertifikat im Kontext weiterer Medienbildungsprozesse gesehen wird.

Medienpädagogik hat auch präventive Aufgaben im Schnittbereich von Medienbildung und dem Kinder- und Jugendmedienschutz wahrzunehmen. Der Schutz von Kindern und Jugendlichen und der Schutz der Menschenwürde sind Rechtsgüter mit Verfassungsrang. Angemessene Maßnahmen des Kinder- und Jugendmedienschutzes sind notwendig. Wir alle wissen, dass gesetzliche Maßnahmen - gerade in Zeiten des Internet - letztlich nur eine begrenzte Wirkung und Reichweite haben. Dies $\mathrm{zu}$ wissen, bedeutet aber nicht, auf sie zu verzichten. Medienpädagogik setzt auf Information, Beratung, Bildung. Es ist notwendig, Eltern und Pädagogen darin zu unterstützen, medienerzieherisches Handeln altersgerecht und situationsangemessen $\mathrm{zu}$ entwickeln. Hierfür sind Formen einer lebensweltorientierten Medienbildung und Medienberatung stärker zu fördern. Dafür reichen allerdings Flyer, Spots im Fernsehen und Internetportale nicht aus.

Um Kinder, Jugendliche, Eltern und Familien in ihren Alltagszusammenhängen zu erreichen und sie zu ermutigen, über eigene Medienpraxen $\mathrm{zu}$ sprechen, bedarf es niedrigschwelliger Zugänge vor Ort. Hierfür braucht es Fachkräfte in Kindergärten, Schulen, Bibliotheken, Jugendeinrichtungen und Jugendverbänden, in der Familien- und Erwachsenenbildung, die über eine medienpädagogische Grundqualifikation verfügen!

Was sind nun die hemmenden Faktoren, die einer nachhaltigen und breitenwirksamen Förderung von Medienkompetenz entgegenstehen?
Diese Frage ist eine der Leitfragen dieses Kongresses. Es genügt nicht, Ziele zu formulieren. Um Maßnahmen zur Umsetzung unserer Ziele auf den Weg zu bringen, ist es wichtig, sich darüber Gedanken zu machen, was die hemmenden Faktoren sind, die bislang einer nachhaltigen und breitenwirksamen Förderung von Medienkompetenz entgegenstehen. Man muss diese Faktoren, diese Schwachstellen kennen, um mit den Maßnahmen richtig ansetzen zu können.

Zur Beantwortung der Frage nach den hemmenden Faktoren habe ich u. a. die 33 Statements von Kolleginnen und Kollegen gelesen, die bislang zur Situation der Medienkompetenzförderung in den Bundesländern auf der Website der Initiative veröffentlicht wurden. Diese Statements ersetzen keine systematische Erhebung zu dieser Frage, sie enthalten aber eine Vielzahl von Faktoren und Hinweisen. Unter Bezug auf diese Statements möchte ich vier Punkte herausstreichen, die mir besonders wichtig erscheinen:

\section{1. „Projektitis“}

Als ein zentraler Faktor wird nahezu in allen Statements genannt,

- dass eine kontinuierliche Projektförderung die Ausnabme ist.

- Die Zeit für permanente Antragstellung und Abrechnung reduziert die Zeit für pädagogische Arbeit und vergeudet Energie.

- In nahezu allen Bereichen fehlt eine medienpädagogische Grundförderung. Eine Verstetigung von erfolgreichen Ideen und Konzepten, eine Nachhaltigkeit von pädagogischen Bemühungen ist so nicht möglich.

- Der Kampf um die begrenzten Gelder erschwert zudem Kooperation und Netzwerkbildung und kostet Zeit, die viel sinnvoller für praktische Arbeit, deren Reflexion und für Weiterbildung investiert werden müsste.

2. An Schulen werden als hemmende Faktoren vor allem genannt:

- Verfestigte und starre Schulstrukturen, viel zu wenig Raum für eigenständige und kreative Formen der Mediennutzung;

- Berufliche Überlastung der Lehrkräfte aufgrund bestehender Lern- und Leistungsschwierigkeiten vieler Schüler und

- eine einseitige Ausrichtung der Schulen auf Leistungsförderung - verstärkt durch den Druck sehr vieler Eltern - werden als hemmende Faktoren gesehen.

- Auch bewahrpädagogische Grundeinstellungen bei vielen Lehrkräften gegenüber 
„den Medien“ erschwert bzw. verhindert Medienbildung.

- Medien werden vielfach - in einem auch didaktisch reduzierten Verständnis - nur als Lernmittel zur Unterrichtsvorbereitung begriffen.

Schule ist in Bezug auf Medien weitgehend noch ein „closed shop“, der sich viel zu wenig ins Gemeinwesen hinein öffnet und außerschulische Erfahrungsfelder einbezieht. Gerade dieser Bereich zeigt: Es geht nicht nur um medienbezogene Fragen - das gesamte System Schule steht auf dem Prüfstand. In diesem Zusammenhang sei auch auf die aktuelle Online-Umfrage "Zukunft durch Bildung" hingewiesen, an der 350.000 Menschen teilnahmen. Die Mehrheit der Befragten bemängelt vor allem die unterschiedlichen Zuständigkeiten der Bundesländer und fordert eine einheitlichere Lösung in schulischen Bildungsfragen.

3. Im Ausbildungsbereich an Hochschulen

- mangelt es an einer Qualifizierung angehender pädagogischer Fachkräfte, sowohl im theoretischen wie im praktischen Bereich.

- Es gibt keine verbindliche Verankerung medienpädagogischer Inhalte und Kompetenzbereiche in den Prüfungs- und Studienordnungen grundständiger pädagogischer Studiengänge.

- Es fehlen Studien- und Ausbildungskapazitäten für eine medienpädagogische Grundbildung.

- An Hochschulen sind Schwankungen über Themen und Ziele zu beobachten; Medienpädagogik hat an den meisten Hochschulen keinen festen Platz, es fehlen koordinierende Stellen.

4. Es fehlt nach wie vor ein politischer und gesellschaftlicher Konsens, Medienbildung als fundamentalen Bestandteil von Bildung in einer Mediengesellschaft zu verstehen.

- Hier hat zwar ein Umdenkungsprozess begonnen; dieser Prozess hat aber noch nicht zur Entwicklung nachhaltiger Förderstrukturen geführt.

- Die nicht vorhandene Grundfinanzierung und Wertschätzung medienpädagogischer Arbeit in nahezu allen Bereichen führt in den Köpfen von sehr vielen Akteuren dazu, dass die Relevanz von Medienkompetenzförderung nicht gesehen wird.

Heute besteht auf dem Kongress in den Arbeitsgruppen und morgen in den beiden Dialogrunden die Gelegenheit, über diese und andere bemmende Faktoren zu diskutieren, die einer breitenwirksamen Medienkompetenzförderung entgegenstehen. Es soll hier nicht be- stritten werden, dass es in Deutschland eine Reihe von Initiativen, Projekten, medienpädagogischen Wettbewerben gibt - wer z. B. die Newsletter der verschiedenen Organisationen liest, kann zum Ergebnis kommen: „Hier ist doch sehr viel geboten!“ Ja, es findet einiges statt. Seit vielen Jahren gibt es Kolleginnen und Kollegen, die unermüdlich arbeiten; auch aus der jüngeren Generation kommen neue Kolleginnen und Kollegen hinzu. Aber nach wie vor haben wir nicht den Zustand erreicht, dass Medienbildung eine Selbstverständlichkeit in der Mediengesellschaft ist.

Während ununterbrochen neue technische Geräte und Möglichkeiten auf den Markt kommen - aktuell steht „cloud computing“ im Vordergrund - und die politischen Eliten dieses Landes auf Fachmessen und Veranstaltungen der Medienwirtschaft präsent sind, scheint sich im politischen Raum bei vielen verantwortlichen Akteuren die Haltung breit zu machen: "Die junge Generation der , digital natives' wird es schon richten. Die junge Leute kommen morgen als Pädagogen in Bildungseinrichtungen und werden dort neuen Schwung reinbringen und auch Medienbildung machen“. Es wird „mehr Gelassenheit" bei Maßnahmen zur Medienkompetenzförderung empfohlen, da doch sehr viele junge Menschen recht kompetent mit Medien umgehen.

Zweifelsohne ist es richtig, dass vermehrt Jahrgänge in die Hochschulausbildung und dann als Lehrkräfte in die Schulen kommen, die mit dem Internet und digitalen Medien aufgewachsen sind. Es gibt durchaus junge Wissenschaftler und Lehrkräfte, die sich engagieren, um Social Media, Web 2.0 und innovative Lehr- und Lernmethoden mit digitalen Medien an Hochschulen und Schulen verstärkt $\mathrm{zu}$ integrieren. Gleichzeitig deuten Studien und Beobachtungen z. B. unter Lehramtsstudierenden und in Schulen darauf hin, dass es nach wie vor eine Kluft zwischen formellen und informellen Lernprozessen mit Medien gibt. Offensichtlich ist es noch nicht gelungen, digitale Medien als Brücke zur Verbindung von formellen und informellen Bildungs- und Lernprozessen hinreichend zu nutzen und entsprechende Potenziale nachhaltig in schulische Bildung zu integrieren. Die Gründe hierfür sind vielschichtig; einer der Gründe ist eine fehlende medienpädagogische Grundbildung aller Lehramtsstudierenden.

Auch in den ca. 60 neuen BachelorStudiengängen zur frübkindlichen Bildung und in Kindergärten und Kindertageseinrichtungen sind wir in Deutschland noch weit entfernt davon, dass Medienbildung fest integriert ist. Ähnlich sieht es im Bereich der sozialpädagogischen Berufe und in der Erwachsenenbildung aus. In der Breite gesehen ist Medienbildung in nahezu allen Ausbildungsgängen und 
in sehr vielen Bildungseinrichtungen einfach noch nicht angekommen. Da reicht es nicht aus, in Dialogrunden über Chancen und Risiken digitaler Medien zu diskutieren, wenn am Ende nicht der politische Wille steht, über neue Projektförderprogramme hinaus endlich an die strukturellen Fragen ranzugehen.

Es ist kontraproduktiv, unter ständigem Hinweis auf die unterschiedlichen Kompetenzbereiche von Bund und Ländern und von verschiedenen Ressorts keine wirksamen Fördermaßnahmen auf den Weg zu bringen, die gerade im Zusammenspiel unterschiedlicher Förder-, Beratungs- und Unterstützungssysteme notwendig sind! Es ist nicht nur die angespannte Haushaltslage in vielen Kommunen, die es nicht einfach macht, Ressourcen für Medienkompetenzförderung aufzubringen und $\mathrm{zu}$ verstetigen - ein nach wie vor existierender Hang zur bildungspolitischen Kleinstaaterei, Ressortdenken, Eitelkeiten bei diversen Akteuren und mangelnde Phantasie verhindern vielerorts Entwicklungen, die möglich wären.

Während die Politik in der Finanz- und Wirtschaftskrise riesige Milliardenbeträge mit Steuergeldern in die Banken und die Wirtschaft gepumpt hat und während die Medienwirtschaft in vielen Bereichen enorme Gewinne macht, bleiben für Medienbildung im Vergleich dazu nur ein paar Krümel übrig. Medien sind zweifelsohne ein wichtiger Wirtschaftsfaktor, sie sind aber auch ein öffentliches Kulturgut. Qualitätsbezogene Medienangebote brauchen Mediennutzer, die Programm- und Medienqualität erkennen und die als Mitglieder dieser Gesellschaft zu befähigen sind, selbst mit Medien kompetent umzugehen. Die Förderung von Medienkompetenz ist für eine demokratische Gesellschaft unverzichtbar! Ich hoffe, dass von diesem Kongress ein deutliches Signal an die Bildungspolitik in Bund und Ländern, an verantwortliche Akteure an Hochschulen und Bildungseinrichtungen ausgeht: Medienpädagogik und Medienbildung dürfen im Bildungsbereich keine Randerscheinung bleiben! Die Förderung der Medienkompetenz von Menschen aus allen Alters- und Sozialgruppen muss zu einem integralen Bestandteil von Bildung werden!

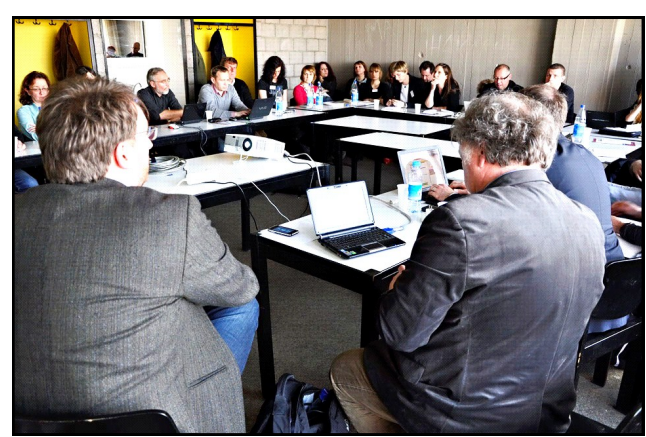

Foto: Vanessa Dreischer-Scheib
Die Positionspapiere für die Beratungen in den Arbeitsgruppen heute Nachmittag belegen eine Vielfalt medienpädagogischer Aufgaben, konzeptioneller Ansätze und Vorschläge für eine nachhaltige Medienkompetenzförderung. Zahlreiche Kolleginnen und Kollegen haben sich im Vorfeld des Kongresses sehr engagiert, um eine aktuelle Einschätzung zur Situation, zu Konzepten und dem erforderlichen Bedarf für eine nachhaltige Förderung in verschiedenen Handlungs- und Themenfeldern zu erarbeiten. Die Grundintentionen des Medienpädagogischen Manifests wurden aufgegriffen, präzisiert und weiter entwickelt. In den Positionspapieren zeigt sich ein großes Potenzial an Ideen, Analysen und Vorschlägen. Bereits die Dokumentation dieser fachlichen Expertise ist ein Erfolg des Kongresses! Ich möchte allen Kolleginnen und Kollegen für dieses Engagement herzlich danken, ein Engagement, das bei allen ehrenamtlich neben den vielfältigen beruflichen Aufgaben erbracht wurde.

Ich wünsche allen Arbeitsgruppen eine intensive und produktive Diskussion. Die Moderatorinnen und Moderatoren der Arbeitsgruppen haben einzelne Kolleginnen und Kollegen zu kurzen Statements gebeten, um bestimmte Themenaspekte zu vertiefen. In der Schlussphase jeder Arbeitsgruppe soll es eine gemeinsame Verständigung über die zentralen bildungspolitischen Vorschläge und Forderungen im jeweiligen Handlungs- und Themenfeld geben. Die zentralen Ergebnisse werden von den Moderatoren in jeder Gruppe festgehalten und morgen allen Kongressteilnehmern in einem Handout zugänglich gemacht.

Morgen, am 2. Kongresstag, bringen dann Kolleginnen zentrale Punkte in AGübergreifender Perspektive in die Podiumsrunden ein, an denen Vertreter von Ministerien, der Kultusministerkonferenz und anderen Bereichen teilnehmen. Die Podiumsrunden werden für eine Plenumsdiskussion geöffnet; der gesamte morgige Tag wird als live-stream im Internet übertragen.

Die Ergebnisse der Arbeitsgruppen und der beiden Dialogrunden werden nach dem Kongress auf der Website der Initiative veröffentlicht. Außerdem ist eine Broschüre geplant, die sich vor allem an bildungspolitische Entscheidungsträger auf Bundes- und Länderebene wendet. Sie sehen: Die Initiative „Keine Bildung ohne Medien!“ ist daran interessiert, dass es auch nach dem Kongress mit dem breiten Bündnis für Medienkompetenzförderung in Deutschland weitergeht. Noch steht nicht fest, in welcher Form die Initiative weiter arbeiten wird. Aber eines ist für mich bereits heute klar: Wir brauchen weiterhin eine wirkungsvolle Vertretung unserer bildungs- und professionspolitischen Anliegen - was hierzu in den bei- 
den letzten Jahren auf den Weg gebracht wurde, sollte Grundlage für weitere Aktivitäten sein!

Ich möchte zum Schluss kommen und mich als Sprecher der Initiative für die große Unterstütrung bedanken, die in den vergangenen Monaten seitens vieler Kolleginnen und Kollegen bei der Vorbereitung des Kongresses, der Arbeitsgruppen und der Netzwerkarbeit erfolgte! Ein besonderer Dank gilt dem Präsidenten der Landesanstalt für Kommunikation Baden-Württemberg, Thomas Langheinrich, und den Mitarbeitern Albrecht Kutteroff und Thomas Rathgeb, sowie dem Direktor der Landesanstalt für Medien Nordrbein-Westfalen, Dr. Jürgen Brautmeier, und den Mitarbeiterinnen Mechthild Appelhoff und Dr. Meike Isenberg. Beide Landesanstalten engagieren sich seit vielen Jahren regional und auch überregional bei der Förderung von innovativen medienpädagogischer Projekten und in der medienpädagogischen Forschung. Die finanzielle Förderung des Kongresses durch die beiden Landesanstalten trug wesentlich dazu bei, dass der Kongress auf solider Basis vorbereitet werden konnte. Dies ist umso bemerkenswerter, als beide Einrichtungen auch zu dem Adressatenkreis der Forderungen des Medienpädagogischen Manifests gehören; wir werten dies als ein sehr ermutigendes Zeichen für die künftige Arbeit - auch im Hinblick auf Kooperationen mit anderen Landesmedienanstalten und Einrichtungen in Deutschland.

Mein Dank gilt auch unserem Kooperationspartner an der Technischen Universität Berlin, Professorin Angela Ittel vom Institut für Erziehungswissenschaft, die durch die Unterstützung des Präsidenten der TU Berlin, Professor Steinbach, u. a. eine kostenfreie Nutzung der Räumlichkeiten an der TU ermöglichte und sich dafür einsetzte, dass nach den zahlreich eingegangenen Anmeldungen weitere Raumkapazitäten zur Verfügung stehen. Last but not least möchte ich mich bei allen Mitgliedern der Koordinationsgruppe, des Projektbüros der Initiative und des lokalen Kongressbüros für die Zusammenarbeit bedanken. Hervorheben möchte ich besonders das Engagement von Alexander Korn, Student im Studiengang Kultur- und Medienbildung an der Pädagogischen Hochschule Ludwigsburg. Alexander engagiert sich seit Herbst 2009 intensiv beim Aufbau und der Pflege der Website der Initiative und übernahm sehr umsichtig und zuverlässig die vielen organisatorischen Aufgaben! Der Dank gilt auch Yvonne Rosendahl, die für das lokale Kongressbüro verantwortlich zeichnet und in den letzten Wochen einige Arbeit zu bewältigen hatte.

Auf dem Hintergrund der zahlreichen Anmeldungen war es unser Ziel, möglichst vielen

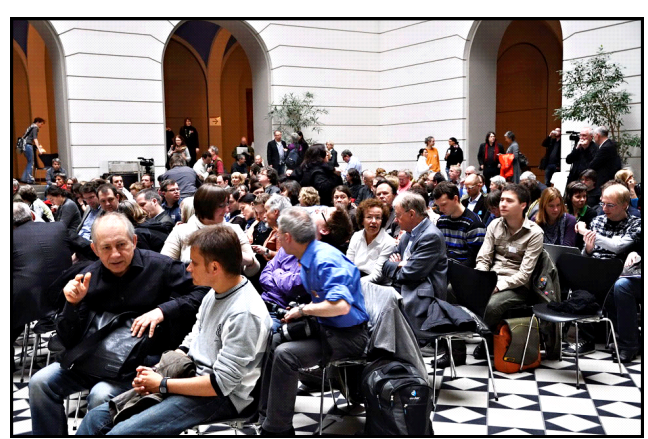

Foto: Vanessa Dreischer-Scheib

ermöglichen. Da kurzfristig für die Plenumsteile kein größerer Hörsaal als der ursprünglich geplante Hörsaal zur Verfügung stand, gibt es eine Videoübertragung der Plenumsteile hier aus dem Lichthof in den nahegelegenen Hörsaal 1012. Wir bitten alle Kongressteilnehmer um die Bereitschaft zur Improvisation und Flexibilität auf dem Kongress.

Ich möchte es nicht versäumen, auf die Posterpräsentationen und die Info-Stände hinzuweisen, die heute Abend ab 18.00 Uhr hier im Lichthof und in den Galerien stattfinden und vielfältige Möglichkeiten zur Information bieten. Ein spezieller Höhepunkt ist dann um 20.30 Uhr die Verleihung des mediusPreises 2011! Der heutige Abend bietet so Gelegenheit zu persönlichen Begegnungen, zum Kennenlernen neuer Ideen und zur Würdigung der Leistung von Studierenden für wissenschaftliche und praxisorientierte $\mathrm{Ab}$ schlussarbeiten.

Alexander Korn wird gleich noch auf ein paar organisatorische Punkte hinweisen, bevor wir eine kleine Pause machen und dann die AGPhase im gegenüberliegenden Gebäude des Instituts für Mathematik beginnt. Ich hoffe auf einen spannenden Kongressverlauf und wünsche Ihnen viele interessante Gespräche, Anregungen und einen angenehmen Aufenthalt in Berlin!

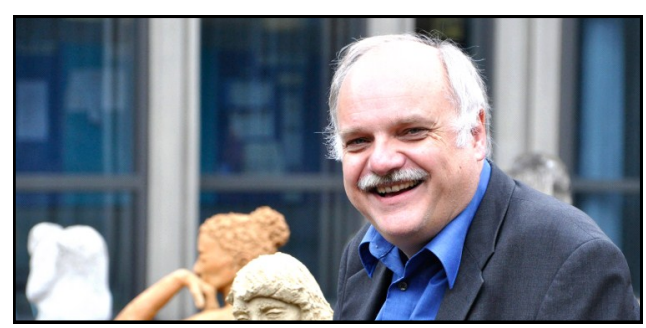

Prof. Dr. Horst Niesyto ist Sprecher der Initiative „Keine Bildung ohne Medien!“ Er ist Professor für Erziehungswissenschaft mit dem Schwerpunkt Medienpädagogik an der Pädagogischen Hochschule Ludwigsburg, Vorsitzender der Sektion Medienpädagogik in der Deutschen Gesellschaft für Erziehungswissenschaft (DGfE) sowie stellv. Mitglied der Kommission für Jugendmedienschutz (KJM). 